\title{
Bounds on weights of nearby cycles and Wakimoto sheaves on affine flag manifolds
}

\author{
Ulrich Görtz and Thomas J. Haines *
}

\begin{abstract}
We study certain nearby cycles sheaves on an affine flag manifold which arise naturally in the Beilinson-Gaitsgory deformation of the affine flag manifold to the affine Grassmannian. We study the multiplicity functions we introduced in an earlier paper, which encode the data of the Jordan-Hoelder series. We prove the multiplicity functions are polynomials in $q$, and we give a sharp bound for their degrees. Our results apply as well to the nearby cycles in the $p$-adic deformation of Laumon-Haines-Ngô, and also to Wakimoto sheaves.
\end{abstract}

\section{Introduction}

Let $G$ be a split connected reductive group over a finite field $\mathbb{F}_{p}$ with algebraic closure $k$, fix an Iwahori subgroup $\mathcal{B} \subset G\left(\mathbb{F}_{p}([\llbracket t])\right)$ and let $\mathcal{F} l=G(k((t))) / \mathcal{B}_{k}$ denote the affine flag variety of $G$. Let $q$ denote a power of $p$ and fix a prime $\ell \neq p$. In [GH], the authors study the Jordan-Hölder series for objects in the Hecke category $P_{q}^{\mathcal{B}}\left(\mathcal{F} l, \overline{\mathbb{Q}}_{\ell}\right)$. This is the category of $\mathcal{B}$-equivariant perverse Weil-sheaves $\mathcal{F}$ on $\mathcal{F} l$ having the property that for any $x \in \mathcal{F} l\left(\mathbb{F}_{q}\right)$, the trace of the Frobenius $\operatorname{Fr}_{q}$ on the stalk $\mathcal{F}_{x}$ satisfies

$$
\operatorname{Tr}\left(\operatorname{Fr}_{q}, \mathcal{F}_{x}\right) \in \mathbb{Z}\left[q, q^{-1}\right]
$$

Put more precisely, for each $x$ there exists a polynomial $P(X, Y) \in \mathbb{Z}[X, Y]$ such that $\operatorname{Tr}\left(\operatorname{Fr}_{q^{n}}, \mathcal{F}_{x}\right)=P\left(q^{n}, q^{-n}\right)$, for all positive integers $n$.

By $[\mathrm{GH}], \S 4$ the objects in $P_{q}^{\mathcal{B}}\left(\mathcal{F l}, \overline{\mathbb{Q}}_{\ell}\right)$ are mixed and have finite length, and the irreducible objects are the integral Tate-twists $I C_{w}(i)$ of the intersection complexes $I C_{w}=j_{w, ! *}\left(\overline{\mathbb{Q}}_{\ell}\right)[\ell(w)]$. Here for $w$ belonging to the extended affine Weyl group $\widetilde{W}$ we take the Goresky-MacPherson middle extension along the immersion $j_{w}: \mathcal{B}_{w} \hookrightarrow \mathcal{B}$ of the corresponding Schubert cell $\mathcal{B}_{w}$. We may thus define non-negative multiplicities $m(\mathcal{F}, w, i)$ by the identity in $P_{q}^{\mathcal{B}}\left(\mathcal{F} l, \overline{\mathbb{Q}}_{\ell}\right)$

$$
\mathcal{F}^{s s}=\bigoplus_{w \in \widetilde{W}} \bigoplus_{i \in \mathbb{Z}} I C_{w}(-i)^{\oplus m(\mathcal{F}, w, i)} .
$$

* Research of the second author partially supported by NSF grant DMS 0303605 and a Sloan Research Fellowship. 
We formally encode the data of the integers $m(\mathcal{F}, w, i)$ with the multiplicity function

$$
m(\mathcal{F}, w):=\sum_{i} m(\mathcal{F}, w, i) q^{i} \in \mathbb{Z}\left[q, q^{-1}\right]
$$

In $[\mathrm{GH}]$ we studied the functions $m(R \Psi, w)$ for certain nearby cycles sheaves $R \Psi$ on $\mathcal{F} l$ which arise as follows. If $G$ is the group $\mathrm{GL}_{n}$ or $\mathrm{GSp}_{2 n}$, there is a $\mathbb{Z}_{p}$-ind-scheme $M$ which is a deformation of the affine Grassmannian Grass $\mathbb{Q}_{p}$ to the affine flag variety $\mathcal{F} l_{\mathbb{F}_{p}}$ for the underlying group $G$. The ind-scheme $M$ has been constructed over $\mathbb{Z}_{p}$ for these two groups in [HN1], and it is related to the bad reduction of certain Shimura varieties (see below). In the function field case the analogous deformation $\mathrm{FL}_{X}$ over a curve $X$ exists for all split connected reductive groups, and has been studied by Gaitsgory [Ga]. In that case, there is a distinguished point $x_{0} \in X$, and the fiber of $\mathrm{FL}_{X}$ over $x \in X$ is $\mathcal{F} l_{k}$ if $x=x_{0}$ and is $\operatorname{Grass}_{k} \times G / B$ if $x \neq x_{0}$, where $G / B$ is the flag variety which is the "reduction modulo $t$ " of $G(k \llbracket t]) / \mathcal{B}_{k}$. Let $\mathcal{Q}_{\mu} \subset$ Grass denote the stratum indexed by a dominant coweight $\mu$ of $G$, and let $I C_{\mu}$ denote the intersection complex $j_{\mu, ! *}\left(\overline{\mathbb{Q}}_{\ell}\right)\left[\operatorname{dim} \mathcal{Q}_{\mu}\right]$. Let $R \Psi_{\mu}=R \Psi\left(I C_{\mu}\right)$, the nearby cycles being taken with respect to the deformation $M$ or $\mathrm{FL}_{X}$. More precisely, in the $p$-adic setting we write $R \Psi_{\mu}=R \Psi^{M}\left(I C_{\mu}\right)$, and in the function-field setting we write $R \Psi_{\mu}=R \Psi^{\mathrm{FL}_{X}}\left(I C_{\mu} \otimes \delta\right)$, where $\delta$ is the skyscraper sheaf supported at the base point of $G / B$.

The sheaf $R \Psi_{\mu}$ belongs to $P_{q}^{\mathcal{B}}\left(\mathcal{F} l, \overline{\mathbb{Q}}_{\ell}\right)$ (see $\left.[\mathrm{GH}], \S 2,6\right)$, a fact that is essentially a consequence of the Kottwitz conjecture, see (4.1). Hence we may consider the multiplicity functions $m\left(R \Psi_{\mu}, w\right)$ for $w \in \widetilde{W}$. By $[\mathrm{GH}], \S 7$ it is known that $m\left(R \Psi_{\mu}, w\right) \neq 0$ if and only if $w \in \operatorname{Adm}(\mu)$, the finite subset of $\mu$-admissible elements in $\widetilde{W}$ (see [KR],[HN2] for explicit descriptions of $\operatorname{Adm}(\mu))$.

Our main theorem is the following result conjectured in $[\mathrm{GH}]$ and proved there for the case $G=\mathrm{GL}_{n}$ or $\mu$ minuscule.

Theorem 1.1 For any $w \in \operatorname{Adm}(\mu)$, the function $m\left(R \Psi_{\mu}, w\right)$ is a polynomial in $q$ having degree at most $\ell(\mu)-\ell(w)$. Equivalently, the same statement holds for the functions $\operatorname{Tr}\left(\operatorname{Fr}_{q},\left(R \Psi_{\mu}\right)_{w}\right)$. Equivalently, for each closed point $z \in \mathcal{B}_{w}$, the weights of the complex $R \Psi_{\mu}\left(\frac{\ell(\mu)}{2}\right)_{z}$ are $\leq \ell(\mu)-\ell(w)$.

Here the length function $\ell: \widetilde{W} \rightarrow \mathbb{Z}_{\geq 0}$ is that defined by the simple affine reflections $S_{\text {aff }}$ through the walls of the alcove in the Bruhat-Tits building which is fixed by the Iwahori $\mathcal{B}$ (see $[\mathrm{GH}])$. The symbol $\ell(\mu)$ denotes the length of the translation element $t_{\mu} \in \widetilde{W}$ corresponding to the cocharacter $\mu$.

The study of $m(R \Psi, w)$ relates to nearby cycles on certain Shimura varieties with Iwahori level structure at $p$. In fact, for such a Shimura variety $S h=S h(\mathbf{G}, h)_{\mathbf{K}}$ where the $p$-adic group $\mathbf{G}_{\mathbb{Q}_{p}}$ is either $\mathrm{GL}_{n} \times \mathbb{G}_{m}$ or $\mathrm{GSp}_{2 n}$, the Rapoport-Zink local model $\mathbf{M}^{\text {loc }}$ for the singularities in the special fiber $S h_{\overline{\mathbb{F}}_{p}}$ can be realized as the scheme-theoretic closure in $M$ of $\mathcal{Q}_{\mu} \subset$ Grass $\mathbb{Q}_{p}$, where $\mu$ is the minuscule coweight coming from the Shimura datum $h$. See [GH], $\S 8$ for a more detailed discussion of the connection with Shimura varieties. 
A related result concerns the Wakimoto sheaves $M_{u, v}$ on $\mathcal{F l}$. Here for elements $u, v \in \widetilde{W}$ we define following I. Mirković the sheaf

$$
M_{u, v}=j_{u !}\left(\overline{\mathbb{Q}}_{\ell}\right)[\ell(u)] \star j_{v *}\left(\overline{\mathbb{Q}}_{\ell}\right)[\ell(v)],
$$

using the standard convolution operation $\star: P_{q}^{\mathcal{B}}\left(\mathcal{F} l, \overline{\mathbb{Q}}_{\ell}\right) \times P_{q}^{\mathcal{B}}\left(\mathcal{F} l, \overline{\mathbb{Q}}_{\ell}\right) \rightarrow D_{c}^{b, \text { Weil }}\left(\mathcal{F} l, \overline{\mathbb{Q}}_{\ell}\right)$, the latter being the category consisting of objects of the "derived category of $\ell$-adic sheaves" $D_{c}^{b}\left(\mathcal{F} l, \overline{\mathbb{Q}}_{\ell}\right)$, equipped with a Weil structure. A result of Mirković states that $M_{u, v}$ is perverse, hence it belongs to $P_{q}^{\mathcal{B}}\left(\mathcal{F} l, \overline{\mathbb{Q}}_{\ell}\right)$ (Mirković's proof appears in $[\mathrm{HP}]$ and in $[\mathrm{AB}]$ ). Let us renormalize and define

$$
\widetilde{M}_{u, v}=M_{u, v}\left(\frac{\ell(u)+\ell(v)-\ell(u v)}{2}\right) .
$$

It is known that the function $\operatorname{Tr}\left(\operatorname{Fr}_{q}, M_{u, v}\right)$ is supported on a subset of the elements $\{x \in$ $\widetilde{W} \mid x \leq u v\}$, where the Bruhat order $\leq$ is defined using $S_{\text {aff }}$ (see [H01]).

Theorem 1.2 For any $w \leq u v$, the function $m\left(\widetilde{M}_{u, v}, w\right)$ is a polynomial in $q$ having degree at most $\ell(u v)-\ell(w)$. Equivalently, the same holds for the functions $\operatorname{Tr}\left(\operatorname{Fr}_{q},\left(\widetilde{M}_{u, v}\right)_{w}\right)$. Equivalently, for each closed point $z \in \mathcal{B}_{w}$, the weights of the complex $\widetilde{M}_{u, v}\left(\frac{\ell(u v)}{2}\right)_{z}$ are $\leq \ell(u v)-\ell(w)$.

As we explain at the end of section 4, Theorem 1.2 can be used to prove Theorem 1.1. But in section 3 we will give a more direct proof of Theorem 1.1 using an approach we introduced (but did not complete) in $[\mathrm{GH}], \S 10$.

Theorem 1.2 might be interesting in its own right, since Wakimoto sheaves have played an important role in several recent papers which study the geometry relating the two presentations (Iwahori-Matsumoto and Bernstein) of an affine Hecke algebra. See [HP], and especially $[\mathrm{AB}]$ and $[\mathrm{ABG}]$.

\section{From bounds on weights to bounds on degrees}

Let $\operatorname{supp}(\mathcal{F})=\left\{x \in \widetilde{W} \mid \operatorname{Tr}\left(\operatorname{Fr}_{q}, \mathcal{F}_{x}\right) \neq 0\right\}$, and let us define the IC-support to be the finite set $\operatorname{ICsupp}(\mathcal{F})=\{x \in \widetilde{W} \mid m(\mathcal{F}, x) \neq 0\}$. An argument in $[\mathrm{GH}], \S 7$ shows that $\operatorname{supp}\left(\widetilde{M}_{u, v}\right) \subseteq$ $\{x \in \widetilde{W} \mid x \leq u v\}=\operatorname{ICsupp}\left(\widetilde{M}_{u, v}\right)$. Using the Kottwitz conjecture (see end of section 4), we get the analogous result for nearby cycles: $\operatorname{supp}\left(R \Psi_{\mu}\right) \subseteq \operatorname{Adm}(\mu)=\operatorname{ICsupp}\left(R \Psi_{\mu}\right)$. For general $\mathcal{F}$, the precise relation between the $\operatorname{sets} \operatorname{supp}(\mathcal{F})$ and $\operatorname{ICsupp}(\mathcal{F})$ is not clear. However, from equation (2.3) below, it is clear that any maximal element of $\operatorname{ICsupp}(\mathcal{F})$ belongs to $\operatorname{supp}(\mathcal{F})$, and vice-versa. Hence the two sets have the same maximal elements.

Let us recall a definition from $[\mathrm{GH}]$. Fix an integer $d$. We say $\mathcal{F}$ satisfies property $(P)_{d}$ if for all $y \in \widetilde{W}$ we have the equivalent identities

$$
\begin{aligned}
\operatorname{Tr}\left(\operatorname{Fr}_{q}, \mathcal{F}_{y}\right) & =\varepsilon_{d} \varepsilon_{y} q^{d} q_{y}^{-1} \overline{\operatorname{Tr}\left(\operatorname{Fr}_{q}, \mathcal{F}_{y}\right)} \\
\operatorname{Tr}\left(\operatorname{Fr}_{q}, D \mathcal{F}_{y}\right) & =\varepsilon_{d} \varepsilon_{y} q^{-d} q_{y}^{-1} \overline{\operatorname{Tr}\left(\operatorname{Fr}_{q}, D \mathcal{F}_{y}\right)},
\end{aligned}
$$

where $\varepsilon_{d}=(-1)^{d}, \varepsilon_{y}=(-1)^{\ell(y)}, q_{y}=q^{\ell(y)}, D \mathcal{F}$ denotes the Verdier dual of $\mathcal{F}$, and $h \mapsto \bar{h}$ is the involution of $\mathbb{Z}\left[q^{1 / 2}, q^{-1 / 2}\right]$ determined by $q^{1 / 2} \mapsto q^{-1 / 2}$. For example, in [GH] we proved that $R \Psi_{\mu}$ satisfies $(P)_{d}$ with $d=\ell(\mu)$ and $\widetilde{M}_{u, v}$ satisfies $(P)_{d}$ with $d=\ell(u v)$. 
Lemma 2.1 Let $\mathcal{F}$ be any object of $P_{q}^{\mathcal{B}}\left(\mathcal{F} l, \overline{\mathbb{Q}}_{\ell}\right)$ and let $d$ be any positive integer. Then for any $x \in \widetilde{W}$, the following statements are equivalent:

(1) For all $w \geq x, \operatorname{Tr}\left(\operatorname{Fr}_{q}, \mathcal{F}_{w}\right)$ is a polynomial in $q$ having degree $\leq d-\ell(w)$;

(2) For all $w \geq x, m(\mathcal{F}, w)$ is a polynomial in $q$ having degree $\leq d-\ell(w)$.

If $\mathcal{F}$ satisfies property $(P)_{d}$, then these are also equivalent to

(3) For all $w \geq x$, the weights of the stalk complex $\mathcal{F}\left(\frac{d}{2}\right)_{w}$ are $\leq d-\ell(w)$.

Proof. For $x, y \in \widetilde{W}$ let $P_{x, y}(q)$ denote the corresponding Kazhdan-Lusztig polynomial. For any $x \in \widetilde{W}$ we have using [KL2] the identity

$$
\operatorname{Tr}\left(\operatorname{Fr}_{q}, \mathcal{F}_{x}\right)=\varepsilon_{x} m(\mathcal{F}, x)+\sum_{w \in \operatorname{ICsupp}(\mathcal{F})} \varepsilon_{w>x} m(\mathcal{F}, w) P_{x, w}(q)
$$

The maximal elements of the $\operatorname{sets} \operatorname{supp}(\mathcal{F})$ and $\operatorname{ICsupp}(\mathcal{F})$ agree, and $(2.3)$ implies the equivalence $(1) \Leftrightarrow(2)$ holds for $x$ a maximal element. The case of general $x$ follows by descending induction with respect to $\leq$, using (2.3) and the bound $\operatorname{deg}_{q} P_{x, w}<\frac{1}{2}(\ell(w)-\ell(x))$ if $x<w$.

Next we will prove that a statement closely related to the implications $(3) \Leftrightarrow(2)$ holds without assuming $\mathcal{F}$ satisifies property $(P)_{d}$. We claim that the following statements are equivalent.

(i) For all $w \geq x$, the weights of $\mathcal{F}\left(\frac{d}{2}\right)_{w}$ are $\leq d-\ell(w)$;

(ii) for all $w \geq x, \operatorname{deg}_{q} m(\mathcal{F}, w) \leq d-\ell(w)$.

Proof of claim. Let us prove $(i) \Rightarrow(i i)$. We fix an element $w \geq x$ and we may assume $w \in \operatorname{ICsupp}(\mathcal{F})$. The condition in (i) holds for $w$ if and only if for all $w^{\prime} \geq w$ and $i \in \mathbb{Z}$ such that $m\left(\mathcal{F}, w^{\prime}, i\right) \neq 0$ we have weight $\left(I C_{w^{\prime}}\left(\frac{\ell\left(w^{\prime}\right)}{2}\right)\left(\frac{d}{2}-\frac{\ell\left(w^{\prime}\right)}{2}-i\right)\right) \leq d-\ell(w)$. Since $I C_{w^{\prime}}\left(\frac{\ell\left(w^{\prime}\right)}{2}\right)$ is a pure weight zero perverse sheaf, this last inequality holds if and only if $2 i-d+\ell\left(w^{\prime}\right) \leq d-\ell(w)$, i.e., $i \leq d-\frac{\ell\left(w^{\prime}\right)+\ell(w)}{2}$. Taking $w^{\prime}=w$, we get $\operatorname{deg}_{q} m(\mathcal{F}, w) \leq d-\ell(w)$, as desired.

Now we prove $(i i) \Rightarrow(i)$. Assume $w \geq x$. The hypothesis means that for every $w^{\prime} \geq w$, and $i$ such that $m\left(\mathcal{F}, w^{\prime}, i\right) \neq 0$ we have $i \leq d-\ell\left(w^{\prime}\right)$ which is a fortiori $\leq d-\frac{\ell(w)+\ell\left(w^{\prime}\right)}{2}$. Now reversing the steps in the paragraph above, we see this implies that weight $\left(\mathcal{F}\left(\frac{d}{2}\right)_{w}\right) \leq d-\ell(w)$. This proves the claim.

In particular we see that (1) and (2) each imply (3) with no extra assumptions on $\mathcal{F}$.

It remains to prove that if $\mathcal{F}$ satisfies $(P)_{d}$ and also satisfies the equivalent conditions (i) and (ii) above, then for all $w \geq x$ the functions $\operatorname{Tr}\left(\operatorname{Fr}_{q}, \mathcal{F}_{w}\right)$ are polynomials in $q$ having degree $\leq d-\ell(w)$. First of all the inequality $\operatorname{deg}_{q} \operatorname{Tr}\left(\operatorname{Fr}_{q}, \mathcal{F}_{w}\right) \leq d-\ell(w)$ follows from (ii) and (2.3). The fact that $\operatorname{Tr}\left(\operatorname{Fr}_{q}, \mathcal{F}_{w}\right)$ is actually a polynomial in $q$ follows from this inequality and the identity $(2.1)$ defining property $(P)_{d}$. 


\section{A bound on the weights of nearby cycles on each stratum}

By Lemma 2.1, Theorem 1.1 can be reformulated as follows.

Theorem 3.1 For each $w \in \operatorname{Adm}(\mu)$ and each closed point $z \in \mathcal{B}_{w}$, the weights of the stalk complex $R \Psi_{\mu}\left(\frac{\ell(\mu)}{2}\right)_{z}$ are $\leq \ell(\mu)-\ell(w)$.

To prove this we will first prove a weaker statement that holds in much greater generality.

\subsection{Bounding weights generically on strata for nearby cycles over Henselian traits}

Let $(S, s, \eta)$ be the spectrum of a complete discrete valuation ring with finite residue field $k(s)$. Let $k(\bar{\eta})$ be a separable closure of the generic point $k(\eta)$, and let $\bar{S}$ be the normalization of $S$ in $\bar{\eta}$, with closed point $\bar{s}$. Let $X$ be an $S$-variety, i.e. an integral separated scheme, flat and of finite type over $S$.

Let $X_{\bar{s}}$ denote the geometric special fiber, and let $D_{c}^{b \text {,Weil }}\left(X, \overline{\mathbb{Q}}_{\ell}\right)$ denote the category consisting of objects in the "derived category" $D_{c}^{b}\left(X_{\bar{s}}, \overline{\mathbb{Q}}_{\ell}\right)$ of $\ell$-adic $\overline{\mathbb{Q}}_{\ell}$-sheaves, equipped with a Weil structure. (See e.g. [GH], §2).

We assume that the special fibre $X_{s}$ of $X$ is stratified by locally closed subschemes $X_{\alpha}$, and that each $X_{\alpha}$ is equidimensional, of dimension $d_{\alpha}$, say.

Definition 3.2 We say that $\mathcal{F} \in D_{c}^{b \text {,Weil }}\left(X, \overline{\mathbb{Q}}_{\ell}\right)$ generically satisfies the sharp bound with respect to $d$, if for each stratum $X_{\alpha}$ there exists an open dense subset $U_{\alpha} \subseteq X_{\alpha}$ such that for all $z \in U_{\alpha}$, the weights on the stalk $\mathcal{F}_{z}$ are $\leq d-\operatorname{dim} X_{\alpha}$.

We say that $\mathcal{F}$ satisfies the sharp bound with respect to $d$ if for each $\alpha$ we may take $U_{\alpha}=X_{\alpha}$.

We say that $\mathcal{F}$ (generically) satisfies the sharp bound on its weights, if it (generically) satisfies the sharp bound w. r. t. $d=\operatorname{dim} X_{s}$.

For any $X / S$ such that $X_{\eta}$ is geometrically integral, we let $I C\left(X_{\eta}\right)$ denote the (perverse) intersection complex of $X_{\eta}$, and we let ${ }^{0} I C\left(X_{\eta}\right)=I C\left(X_{\eta}\right)\left(\frac{\operatorname{dim} X_{\eta}}{2}\right)$ denote the Tate-twist of $I C\left(X_{\eta}\right)$ that is a self-dual and weight zero perverse sheaf on $X_{\eta}$. Finally, we define the "normalized" nearby cycles complex in $D_{c}^{b \text {,Weil }}\left(X, \overline{\mathbb{Q}}_{\ell}\right)$ by

$$
{ }^{0} R \Psi\left(I C\left(X_{\eta}\right)\right)=R \Psi^{X}\left({ }^{0} I C\left(X_{\eta}\right)\right) .
$$

It is well-known that nearby cycles preserve (middle) perversity, and so ${ }^{0} R \Psi\left(I C\left(X_{\eta}\right)\right)$ is an object of $P_{\text {Weil }}\left(X, \overline{\mathbb{Q}}_{\ell}\right)$, the full subcategory $D_{c}^{b, \text { Weil }}\left(X, \overline{\mathbb{Q}}_{\ell}\right)$ whose objects are perverse.

Note that it would be more correct to say that the nearby cycles is an object of the category $D_{c}^{b}\left(X \times{ }_{s} \eta, \overline{\mathbb{Q}}_{\ell}\right)$, or of its subcategory $P\left(X \times{ }_{s} \eta, \overline{\mathbb{Q}}_{\ell}\right)$ of perverse objects (see e.g. [GH], $\left.\S 2,5\right)$. But as in loc. cit., by choosing a lift $\operatorname{Fr}_{q} \in \operatorname{Gal}(k(\bar{\eta}) / k(\eta))$ of the geometric Frobenius generator in $\operatorname{Gal}(k(\bar{s}) / k(s))$, we can and will regard the nearby cycles as being an object in $P_{\text {Weil }}\left(X, \overline{\mathbb{Q}}_{\ell}\right)$. This causes no problems in the statement or application of the proposition below. 
Proposition 3.3 Let $X / S$ be an $S$-variety such that $X_{\eta}$ is geometrically integral. Suppose that the special fiber $X_{s}$ is stratified by locally closed subvarieties $X_{\alpha}$ which are equidimensional of dimension $d_{\alpha}$. Then ${ }^{0} R \Psi\left(I C\left(X_{\eta}\right)\right)$ generically satisfies the sharp bound on its weights.

Proof. By the calculations of Rapoport-Zink [RZ], the sharp bound holds in the case where $X / S$ is proper strictly semi-stable, in the terminology of de Jong [deJ]. We will reduce the proposition to that case.

The key ingredient in the reduction is the following result of de Jong ([deJ], Theorem 6.5).

Theorem 3.4 (de Jong) Let $X / S$ be an $S$-variety. Then there exists a trait $S^{\prime}=\left(S^{\prime}, s^{\prime}, \eta^{\prime}\right)$ finite over $S$, an $S^{\prime}$-variety $X^{\prime}$ for which there is an alteration $X^{\prime} \rightarrow X$ of $S$-varieties, and an open immersion $j: X^{\prime} \hookrightarrow \overline{X^{\prime}}$ of $S^{\prime}$-varieties such that $\overline{X^{\prime}}$ is a proper strictly semi-stable $S^{\prime}$-variety:

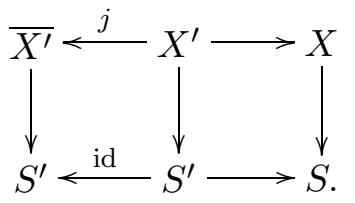

We will make use of this theorem in exactly the same manner as $[\mathrm{GH}], \S 10$, where we proved that ${ }^{0} R \Psi\left(I C\left(X_{\eta}\right)\right)$ is mixed.

Let $r: X_{S^{\prime}} \rightarrow X$ be the projection morphism defined by the diagram in Theorem 3.4. Note that ${ }^{0} I C\left(X_{\eta^{\prime}}\right)={ }^{0} I C\left(X_{\eta}\right)_{\eta^{\prime}}$. By invariance of nearby cycles under change of traits ([SGA

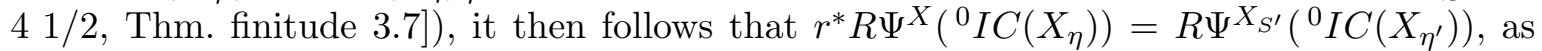
objects of $P_{\text {Weil }}\left(X_{S^{\prime}}, \overline{\mathbb{Q}}_{\ell}\right)$. We have used here that $X_{S^{\prime}}$ and $X$ have "the same" geometric special fiber $X_{\bar{s}}$, since $k(s)$ is finite. Thus, to bound the weights of ${ }^{0} R \Psi\left(I C\left(X_{\eta}\right)\right)$ generically

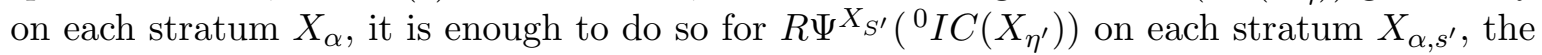
base-change of $X_{\alpha}$ via $s^{\prime} \rightarrow s$.

We may therefore replace $X / S$ with $X_{S^{\prime}} / S^{\prime}$. In effect, this means we may assume $S^{\prime}=S$ and that we have an alteration $\pi: X^{\prime} \rightarrow X$ of $S$-varieties and an open immersion $j: X^{\prime} \hookrightarrow \overline{X^{\prime}}$ of $S$-varieties such that $\overline{X^{\prime}} / S$ is a proper strictly semi-stable $S$-variety.

Let us denote simply by $\pi$ the induced morphisms $\pi: X_{\eta}^{\prime} \rightarrow X_{\eta}$ and $\pi: X_{s}^{\prime} \rightarrow X_{s}$ on generic and special fibers, and on the corresponding geometric fibers. For any object $\mathcal{K}$ of $D_{c}^{b}\left(X_{\bar{\eta}}, \overline{\mathbb{Q}}_{\ell}\right)$ or $D_{c}^{b}\left(X_{\bar{s}}, \overline{\mathbb{Q}}_{\ell}\right)$, let ${ }^{p} \mathrm{H}^{i} \mathcal{K}\left(\right.$ resp. $\left.\mathrm{H}^{i} \mathcal{K}\right)$ denote the $i$-th cohomology sheaf of $\mathcal{K}$ for the perverse (resp. standard) $t$-structure. Also, let ${ }^{p} \tau^{\geq i}$ be the perverse truncation functor. Let ${ }^{p} \pi_{*}:={ }^{p} \mathrm{H}^{0} \pi_{*}$ denote the perverse version of the push-forward functor $\pi_{*}:=R \pi_{*}$ on derived categories. Since $\pi$ is proper, $\pi_{*}=\pi_{!}$and hence ${ }^{p} \pi_{*}={ }^{p} \pi_{!}$.

Let $\Lambda_{\eta}^{X^{\prime}}$ denote the constant sheaf $\overline{\mathbb{Q}}_{\ell}$ on $X_{\eta}^{\prime}$. For $n=\operatorname{dim}\left(X_{\eta}\right)$ let $A^{X^{\prime}}=\Lambda_{\eta}^{X^{\prime}}[n]\left(\frac{n}{2}\right)$. The smoothness of $X_{\eta}^{\prime}$ means that $A^{X^{\prime}}$ is a self-dual perverse sheaf on $X_{\eta}^{\prime}$ which is pure of weight 0 .

In this circumstance, we know by $[\mathrm{GH}]$, Lemma 10.7 , that ${ }^{0} I C\left(X_{\eta}\right)$ is a subquotient of the perverse sheaf ${ }^{p} \pi_{*}\left(A^{X^{\prime}}\right)$. By the exactness of the functor $R \Psi^{X}$, the perverse 
sheaf $R \Psi^{X}\left({ }^{0} I C\left(X_{\eta}\right)\right)$ is a subquotient of $R \Psi^{X}\left({ }^{p} \pi_{*} A^{X^{\prime}}\right)={ }^{p} \pi_{*} R \Psi^{X^{\prime}}\left(A^{X^{\prime}}\right)$ in the category $P_{\text {Weil }}\left(X, \overline{\mathbb{Q}}_{\ell}\right)$. Thus, it is enough to prove the sharp bound holds for the complex ${ }^{p} \pi_{!} R \Psi^{X^{\prime}}\left(A^{X^{\prime}}\right)_{z}$, whenever $z \in U_{\alpha}$ for some open dense subset $U_{\alpha} \subseteq X_{\alpha}$.

To bound the weights of ${ }^{p} \pi_{!} R \Psi^{X^{\prime}}\left(A^{X^{\prime}}\right)_{z}$, we claim it is enough to bound the weights of $\pi_{!} R \Psi^{X^{\prime}}\left(A^{X^{\prime}}\right)_{z}$. To see this, note that $\pi_{!} A^{X^{\prime}}$ is a pure complex of "geometric origin" on $X_{\eta}$, in the terminology of $[\mathrm{BBD}], \S 5,6$. Hence for each $i$ the distinguished triangle

$$
{ }^{p} \mathrm{H}^{i} \pi ! A^{X^{\prime}} \longrightarrow{ }^{p}{ }^{\geq i} \pi ! A^{X^{\prime}} \longrightarrow{ }^{p}{ }^{\geq i+1} \pi ! A^{X^{\prime}}
$$

becomes a direct sum over $\bar{\eta}$ (loc. cit. Thm. 5.4.5, 6.2.5). Applying the functor $R \Psi^{X^{\prime}}(\cdot)_{z}$ we get a distinguished triangle

$$
{ }^{p} \mathrm{H}^{i} \pi_{!} R \Psi^{X^{\prime}}\left(A^{X^{\prime}}\right)_{z} \longrightarrow{ }^{p} \tau^{\geq i} \pi ! R \Psi^{X^{\prime}}\left(A^{X^{\prime}}\right)_{z} \longrightarrow{ }^{p^{\geq i+1}} \pi ! R \Psi^{X^{\prime}}\left(A^{X^{\prime}}\right)_{z}
$$

which becomes a direct sum once we forget the Galois actions. Thus, for each $j$ we have a short exact sequence of $\ell$-adic Galois modules

$$
0 \longrightarrow \mathrm{H}^{j}\left({ }^{p} \mathrm{H}^{i} \mathcal{K}_{z}\right) \longrightarrow \mathrm{H}^{j}\left({ }^{p} \tau^{\geq i} \mathcal{K}_{z}\right) \longrightarrow \mathrm{H}^{j}\left({ }^{p} \tau^{\geq i+1} \mathcal{K}_{z}\right) \longrightarrow 0
$$

where for brevity we have written $\mathcal{K}_{z}$ in place of $\pi_{!} R \Psi^{X^{\prime}}\left(A^{X^{\prime}}\right)_{z}$. Note that for $i \ll 0$ the middle term is $\mathrm{H}^{j}\left(\pi_{!} R \Psi^{X^{\prime}}\left(A^{X^{\prime}}\right)_{z}\right)$. It now follows easily by ascending induction on $i$ that if the sharp bound generically holds for the weights of $\pi_{!} R \Psi^{X^{\prime}}\left(A^{X^{\prime}}\right)_{z}$ then the sharp bound generically holds for each ${ }^{p} \mathrm{H}^{i} \pi_{!} R \Psi^{X^{\prime}}\left(A^{X^{\prime}}\right)_{z}$, and in particular for ${ }^{p} \pi ! R \Psi^{X^{\prime}}\left(A^{X^{\prime}}\right)_{z}$.

Now returning to the diagram in Theorem 3.4, let $\Lambda_{\eta}^{\overline{X^{\prime}}}$ denote the constant sheaf $\overline{\mathbb{Q}}_{\ell}$ on $\overline{X^{\prime}}{ }_{\eta}$, with corresponding perverse Tate-twist $A^{\overline{X^{\prime}}}:=\Lambda_{\eta}^{\overline{X^{\prime}}}[n]\left(\frac{n}{2}\right)$. Let $D_{i}$ be the irreducible components on the special fiber of $X^{\prime}$. The formula

$$
j^{*} R \Psi^{\overline{X^{\prime}}}\left(A^{\overline{X^{\prime}}}\right)=R \Psi^{X^{\prime}}\left(A^{X^{\prime}}\right)
$$

and the results of Rapoport-Zink [RZ] for the proper strictly semi-stable model $\overline{X^{\prime}} \rightarrow S$ show that the weights of $R \Psi^{X^{\prime}}\left(A^{X^{\prime}}\right)$ on any intersection $\cap_{i \in J} D_{i}$ are $\leq \operatorname{dim}\left(X_{\bar{s}}^{\prime}\right)-\operatorname{dim}\left(\cap_{i \in J} D_{i}\right)$. Here $J$ denotes any finite subset of the set indexing the components $D_{i}$.

Now we can complete the proof of the proposition. Since $\pi: X_{s}^{\prime} \rightarrow X_{s}$ is proper, the union of the sets $X_{\alpha} \cap \pi\left(\cap_{i \in I} D_{i}\right)$, where $I$ ranges over all subsets of indices such that $\operatorname{dim}\left(\cap_{i \in I} D_{i}\right)<d_{\alpha}$, is a proper closed subset of $X_{\alpha}$. Moreover, since $X_{\alpha}$ is equidimensional the complement $U_{\alpha} \subseteq X_{\alpha}$ of this closed subset is open and dense. For $z \in U_{\alpha}$ the fiber $\pi^{-1}(z)$ does not meet any intersection of irreducible components in $X_{s}^{\prime}$ of form $\cap_{i \in I} D_{i}$ whose dimension is $<d_{\alpha}$. It follows that the weights of $R \Psi^{X^{\prime}}\left(A^{X^{\prime}}\right)$ on the fiber $\pi^{-1}(z)$ are $\leq \operatorname{dim}\left(X_{\bar{s}}^{\prime}\right)-d_{\alpha}$, by the above remarks. Hence for $z \in U_{\alpha}$ the weights of $\pi_{!} R \Psi^{X^{\prime}}\left(A^{X^{\prime}}\right)_{z}=R \Gamma_{c}\left(\pi^{-1}(z), R \Psi^{X^{\prime}}\left(A^{X^{\prime}}\right)\right)$ are $\leq \operatorname{dim}\left(X_{\bar{s}}^{\prime}\right)-d_{\alpha}$, and we are done.

\subsection{Application to the affine flag manifold}

Of course, the generic sharp bound on the weights is most interesting in cases where the weights are actually constant along the strata, in particular when one is dealing with sheaves 
equivariant under some group action, where the stratification is given by the orbits. This is the situation we considered in $[\mathrm{GH}]$ : in either the $p$-adic or the function-field setting, the sheaves $R \Psi_{\mu}$ we consider are $\mathcal{B}$-equivariant hence constant along the $\mathcal{B}$-orbits $\mathcal{B}_{w}$, for $w \in \widetilde{W}$; see loc. cit. $\S 2$ and [HN1],[Ga]. Hence we have the following corollary of Proposition 3.3, which immediately proves Theorems 3.1 and 1.1 .

Corollary 3.5 Since $R \Psi_{\mu}\left(\frac{\ell(\mu)}{2}\right)$ is $\mathcal{B}$-equivariant, it satisfies the sharp bound on its weights with respect to $d=\ell(\mu)=\operatorname{dim}\left(\mathcal{Q}_{\mu}\right)$ and the stratification by $\mathcal{B}$-orbits.

\subsection{The case where $R \Psi$ is smooth along the strata}

The referee for this article suggested that we include the following consequence of Proposition 3.3. We thank the referee for his remarks.

We say a mixed complex $\mathcal{F} \in D_{c}^{b \text {,Weil }}\left(X, \overline{\mathbb{Q}}_{\ell}\right)$ is smooth along a stratum $X_{\alpha}$ if for all $i$ the restriction of the cohomology sheaf $\mathrm{H}^{i} \mathcal{F}$ is a smooth (= lisse) Weil sheaf on $X_{\alpha}$.

Proposition 3.6 In the situation of Proposition 3.3, assume that $R \Psi\left(I C\left(X_{\eta}\right)\right)$ is smooth along every stratum $X_{\alpha}$. Then ${ }^{0} R \Psi\left(I C\left(X_{\eta}\right)\right)$ satisfies the sharp bound on its weights, with respect to this stratification.

Proof. This follows immediately from Proposition 3.3 and the semicontinuity of weights theorem (e.g. $[\mathrm{KW}]$, Theorem I.2.8), applied to the smooth Weil sheaves $\left.{ }^{0} R^{i} \Psi\left(I C\left(X_{\eta}\right)\right)\right|_{X_{\alpha}}$.

We remark that this proposition does not allow us to avoid invoking that $R \Psi_{\mu}$ is $\mathcal{B}$-equivariant in the proof of Corollary 3.5, because a priori we know only that $R \Psi_{\mu}$ is constructible along the $\mathcal{B}$-orbits. We could of course replace the stratification by $\mathcal{B}$-orbits with a finer stratification along whose strata $R \Psi_{\mu}$ definitely is smooth. But since Proposition 3.6 would pertain only to the finer stratification, the end result could be weaker than what we assert in Corollary 3.5 .

\section{Bounding degrees of coefficients in Wakimoto functions}

In this section we give a combinatorial result which proves Theorem 1.2 and which also yields — via the Kottwitz conjecture (4.1) - a different proof of Theorem 1.1.

By Lemma 2.1, Theorem 1.2 can be reformulated as follows.

Theorem 4.1 For each $w \leq u v$, the function $\operatorname{Tr}\left(\operatorname{Fr}_{q},\left(\widetilde{M}_{u, v}\right)_{w}\right)$ is a polynomial in $q$ having degree $\leq \ell(u v)-\ell(w)$. 
We will need some further notation. Recall we consider a split connected reductive group $G$ over a field $k$. Let us fix a choice of Borel subgroup $B \subset G$ and maximal torus $T \subset B$. Let $\mathcal{B} \subset G(k \llbracket t])$ be the Iwahori subgroup whose "reduction modulo $t$ " is $B$. The data $(G, B, T)$ determine a (based) root system and a set of simple reflections $S$ generating the Coxeter group $(W, S)$, where $W=N_{G} T / T$ is the finite Weyl group. Let $w_{0}$ denote the unique longest element in $W$. Let us choose an origin in the apartment corresponding to $T$ and a Weyl chamber $\operatorname{Ch}(B)$ corresponding to $B$. Let $A$ denote the base alcove, that is, the unique alcove in the given apartment whose closure contains the origin and which lies in $\operatorname{Ch}(B)$. Let $\bar{B}$ be the unique opposite Borel subgroup, that is, the one such that $B \cap \bar{B}=T$.

Let $W_{\text {aff }}$ denote the affine Weyl group of $G$. It is a Coxeter group with generators $S_{\text {aff }}$, the set of simple reflections through the walls of the opposite alcove $w_{0}(A)$ of the base alcove $A$ : if $G$ is almost simple with $B$-positive simple roots $\alpha_{i}(1 \leq i \leq l)$ and $B$-highest root $\widetilde{\alpha}$, then $w\left(A_{0}\right)$ is the alcove

$$
w_{0}(A)=\left\{x \in X_{*}(T) \otimes \mathbb{R} \mid 0<\left\langle-\alpha_{i}, x\right\rangle<1 \text { for all } i \text {, and }\langle-\widetilde{\alpha}, x\rangle<1\right\} .
$$

In this case the simple affine reflections are the reflections $s_{-\alpha_{i}}=s_{\alpha_{i}}$ and $s_{0}:=s_{\widetilde{\alpha}+1}=$ $t_{-\widetilde{\alpha}^{\vee}} s_{\widetilde{\alpha}}$. (See also $[\mathrm{GH}]$, section 2.2.)

The alcove fixed by $\mathcal{B}$ is the opposite alcove $w_{0}(A)$. This results from our convention that we embed $\widetilde{W}$ into $G(k((t)))$ using the identification of $\nu \in X_{*}(T)$ with $\nu(t) \in G(k((t)))$.

The $\bar{B}$-positive affine roots $\alpha+k$ are those taking positive values on $w_{0}(A)$. Equivalently, $\alpha+k$ is $\bar{B}$-positive if and only if either $k \geq 1$, or $k=0$ and $\alpha$ is $\bar{B}$-positive.

The set of affine roots carries a left action by the group $\widetilde{W}$ by the rule

$$
x \cdot(\alpha+k)=(\alpha+k) \circ x^{-1} .
$$

Lemma 4.2 Let $x \in \widetilde{W}$ and suppose $\alpha+k$ is a $\bar{B}$-positive affine root, with corresponding reflection $s=s_{\alpha+k}$. Let $\leq$ denote the Bruhat order on $\widetilde{W}$ defined using $S_{\text {aff. Then }}$

(a) $x<x s$ if and only if $x \cdot(\alpha+k)$ is $\bar{B}$-positive;

(b) $x<s x$ if and only if $x^{-1} \cdot(\alpha+k)$ is $\bar{B}$-positive.

Proof. It is enough to prove (b). Write $A^{-}:=w_{0}(A)$. We note that $x<s x$ if and only if $x A^{-}$and $A^{-}$lie on the same side of the hyperplane $\alpha+k=0$, which happens if and only if $(\alpha+k) \circ x$ takes positive values on $A^{-}$, i.e., $x^{-1} \cdot(\alpha+k)$ is $\bar{B}$-positive.

We will also need one more lemma.

Lemma 4.3 Suppose $t=s_{\beta}$, where $\beta$ is a $\bar{B}$-positive affine root. Let $u, v \in \widetilde{W}$ and suppose $u<u t$. Then

$$
u v<u t v \Longleftrightarrow v<t v .
$$


Proof. Note first that $u t u^{-1}=s_{u \cdot \beta}$ and $u \cdot \beta$ is $\bar{B}$-positive, by Lemma 4.2. Then we have

$$
\begin{aligned}
u v<u t v & \Longleftrightarrow u v<s_{u \cdot \beta} u v \\
& \Longleftrightarrow(u v)^{-1} \cdot(u \cdot \beta) \text { is } \bar{B} \text {-positive } \\
& \Longleftrightarrow v^{-1} \cdot \beta \text { is } \bar{B} \text {-positive } \\
& \Longleftrightarrow v<t v
\end{aligned}
$$

again by Lemma 4.2 .

For elements $u, v \in \widetilde{W}$, we consider the Wakimoto functions $\widetilde{T}_{u} \widetilde{T}_{v^{-1}}^{-1}$, see [GH], section 7 . Here for $x \in \widetilde{W}$, the symbol $T_{x}$ denotes the standard generator $\operatorname{char}(\mathcal{B} \mathrm{x} \mathcal{B})$ in the Iwahori-Hecke algbra $C_{c}\left(\mathcal{B} \backslash G\left(\mathbb{F}_{q}((t))\right) / \mathcal{B}\right)$ and $\widetilde{T}_{x}:=q_{x}^{-1 / 2} T_{x}$. Our goal is to prove the following improved version of loc. cit. Proposition 7.6 (where the same conclusion is obtained under the additional hypothesis that $\widetilde{T}_{u} \widetilde{T}_{v^{-1}}^{-1}$ has a minimal expression). The indeterminate $Q$ that appears here is defined by the relation $q^{1 / 2} Q=1-q$.

Proposition 4.4 If $\widetilde{T}_{u} \widetilde{T}_{v^{-1}}^{-1}=\sum_{x} R_{x, v}^{u}(Q) \widetilde{T}_{x}$, then $R_{x, v}^{u}(Q)$ is a polynomial in $Q$ with integer coefficients, and

$$
\operatorname{deg}_{Q} R_{x, v}^{u}(Q) \leq \ell(u v)-\ell(x)
$$

Proof. We may assume $u, v \in W_{\text {aff }}$. Consider reduced expressions $u=t_{1} \cdots t_{k}$ and $v=$ $s_{1} \cdots s_{r}$, where $t_{j}, s_{i}$ belong to $S_{\text {aff }}$. Write $\tilde{t}_{j}$ resp. $\tilde{s}_{i}^{-1}$ as short-hand for $\widetilde{T}_{t_{j}}$ resp. $\widetilde{T}_{s_{i}}^{-1}$. We will prove the desired bound

$$
\operatorname{deg}_{Q} \tilde{t}_{1} \cdots \tilde{t}_{k} \cdot \tilde{s}_{1}^{-1} \cdots \tilde{s}_{r}^{-1}(x) \leq \ell(u v)-\ell(x),
$$

by induction on $k$, the case $k=0$ being obvious. We assume the bound holds for $k \geq 0$ and deduce it for $k+1$.

Let $t=s_{\beta}$, where $\beta$ is a $\bar{B}$-positive simple affine root, and suppose $u<u t=t_{1} \cdots t_{k} t$.

Case 1: We have $v<t v$ (so by Lemma 4.3, uv<utv). Then because $\tilde{t}^{-1}=\tilde{t}+Q$, we have

$$
\tilde{t}_{1} \cdots \tilde{t}_{k} \tilde{t}_{1}^{-1} \cdots \tilde{s}_{r}^{-1}(x)=\tilde{t}_{1} \cdots \tilde{t}_{k} \tilde{t}^{-1} \tilde{s}_{1}^{-1} \cdots \tilde{s}_{r}^{-1}(x)-Q \tilde{t}_{1} \cdots \tilde{t}_{k} \tilde{s}_{1}^{-1} \cdots \tilde{s}_{r}^{-1}(x) .
$$

Now by induction the $\operatorname{deg}_{Q}$ of the first term on the right is bounded by $\ell(u t v)-\ell(x)$. Similarly the $\operatorname{deg}_{Q}$ of the second term is bounded by $1+\ell(u v)-\ell(x) \leq \ell(u t v)-\ell(x)$.

Case 2: We have $t v<v$ (so by Lemma 4.3, utv $<u v$ ). Then we can write a reduced expression for $v$ in the form $v=t s_{2} \cdots s_{r}$, and thus we are considering

$$
\tilde{t}_{1} \cdots \tilde{t}_{k} \tilde{t}^{-1} \tilde{s}_{2}^{-1} \cdots \tilde{s}_{r}^{-1}(x)=\tilde{t}_{1} \cdots \tilde{t}_{k} \tilde{s}_{2}^{-1} \cdots \tilde{s}_{r}^{-1}(x)
$$

and clearly our induction hypothesis implies that the $\operatorname{deg}_{Q}$ is bounded by $\ell(u t v)-\ell(x)$, as desired.

The fact that $R_{x, v}^{u}$ is a polynomial in $Q$ with integer coefficients can also be proved by induction on $k=\ell(u)$ in a similiar way, or by using the Iwahori-Matsumoto relations in the Hecke algebra (see [H01]). 
Let us now state explicitly what Proposition 4.4 says about the weights of the Wakimoto sheaves $\widetilde{M}_{u, v}$. Recall that for $u, v \in \widetilde{W}$, the Wakimoto sheaf is defined as

$$
M_{u, v}=j_{u !} \overline{\mathbb{Q}}_{\ell}[\ell(u)] \star j_{v *} \overline{\mathbb{Q}}_{\ell}[\ell(v)],
$$

where $j_{u}, j_{v}$ are the inclusions of the Schubert cells corresponding to $u, v$ into the affine flag variety, where $j_{u !}, j_{v *}$ denote the derived functors, and where $\star$ is the convolution product of perverse sheaves. The function associated to $M_{u, v}$ via the sheaf-function dictionary is

$$
\varepsilon_{u} \varepsilon_{v} q_{v} T_{u} T_{v^{-1}}^{-1}=\varepsilon_{u} \varepsilon_{v} q_{u}^{1 / 2} q_{v}^{1 / 2} \sum_{x} q_{x}^{-1 / 2} R_{x, v}^{u} T_{x}
$$

and so the function associated to $\widetilde{M}_{u, v}$ is $\varepsilon_{u} \varepsilon_{v} q_{u v}^{1 / 2} \sum_{x} q_{x}^{-1 / 2} R_{x, v}^{u} T_{x}$.

From $[\mathrm{GH}], \S 7$ we know that for any power $Q^{i}$ which appears with non-zero coefficient in $R_{x, v}^{u}(Q)$, the integer $i$ has the same parity as $\ell(u v)-\ell(x)$. This together with the identity $q^{1 / 2} Q=1-q$ and the bound $\operatorname{deg}_{Q} R_{x, v}^{u}(Q) \leq \ell(u v)-\ell(x)$ of Proposition 4.4 gives the following result, which is a reformulation of Theorems 4.1 and 1.2.

Corollary 4.5 Let $u, v, x \in \widetilde{W}$. Then $\operatorname{Tr}\left(\operatorname{Fr}_{q},\left(\widetilde{M}_{u, v}\right)_{x}\right)$ is a polynomial in $q$ of degree $\leq$ $\ell(u v)-\ell(x)$. The sheaf $\widetilde{M}_{u, v}\left(\frac{\ell(u v)}{2}\right)$ satisfies the sharp bound with respect to $d=\ell(u v)$ and the stratification by $\mathcal{B}$-orbits.

We remark that via the Kottwitz conjecture stated below, this corollary can be used to give another proof of Theorem 1.1. The Kottwitz conjecture is the following identity in the IwahoriHecke algebra $C_{c}\left(\mathcal{B} \backslash G\left(\mathbb{F}_{q}((t))\right) / \mathcal{B}\right)$. In the function-field setting it was proved by Gaitsgory [Ga], whose ideas were adapted to prove it in the $p$-adic setting in [HN1].

$$
\operatorname{Tr}\left(\operatorname{Fr}_{q}, R \Psi_{\mu}\right)=\varepsilon_{\mu} q_{\mu}^{1 / 2} \sum_{\lambda \in \Omega(\mu)} m_{\mu}(\lambda) \Theta_{\lambda}
$$

The original form concerned the semi-simple trace of Frobenius, but by virtue of the unipotence of the inertia action on $R \Psi_{\mu}$ which was proved by Gaitsgory [Ga] (see also [GH] $\S 5$ ), we may replace the semi-simple trace $\operatorname{Tr}^{s s}$ with the usual trace, once we have chosen a lift $\mathrm{Fr}_{q}$ of geometric Frobenius, as we have already done. See the discussion of this point in $[\mathrm{GH}]$, $\S 5$.

Let us explain the notation on the right hand side. We let $\Omega(\mu)$ denote the set of coweights $\lambda \in X_{*}(T)$ such that for every $w \in W, \mu-w \lambda$ is a sum of $B$-positive coroots. For such a coweight $\lambda$, write it as $\lambda=\lambda_{1}-\lambda_{2}$, where $\lambda_{i}$ is $B$-dominant for $i=1,2$. Then following Bernstein we define

$$
\Theta_{\lambda}=\widetilde{T}_{t_{\lambda_{1}}} \widetilde{T}_{t_{\lambda_{2}}}^{-1}
$$

Let $G^{\vee}$ be the dual group for $G$. The number $m_{\mu}(\lambda)$ is the multiplicity with which the weight $\lambda$ appears in the character $E_{\mu}$ of the simple $G^{\vee}$-module with highest weight $\mu$.

Note that $\ell(\lambda) \leq \ell(\mu)$ and the difference $\ell(\mu)-\ell(\lambda)$ is an even integer. It is now clear that Theorem 1.1 can be deduced, via Lemma 2.1, by applying Corollary 4.5 with $u=t_{\lambda_{1}}$ and $v=t_{-\lambda_{2}}$ for all $\lambda \in \Omega(\mu)$ and by using (4.1). 


\section{References}

[AB] S. Arkhipov, R. Bezrukavnikov, Perverse sheaves on affine flags and Langlands dual group, Preprint math.RT/0201073 (2002).

[ABG] S. Arkhipov, R. Bezrukavnikov, V. Ginzburg, Quantum groups, the loop Grassmannian, and the Springer resolution, J. Amer. Math. Soc. 17 (2004), 595-678.

[BBD] A. Beilinson, I.N. Bernstein, P. Deligne, Faisceaux Pervers, Astérisque 100, (1981).

[deJ] A. de Jong, Smoothness, semi-stability and alterations, Publ. Math. I.H.E.S, 83, (1996) 51-93.

[Ga] D. Gaitsgory. Construction of central elements in the affine Hecke algebra via nearby cycles. Invent. Math. 144 (2001), 253-280.

[GH] U. Görtz, T. Haines, The Jordan-Hölder series for nearby cycles on some Shimura varieties and affine flag varieties, Preprint Bonn 2004, math.AG/0402143.

[H01] T. Haines, The combinatorics of Bernstein functions, Trans. Amer. Math. Soc. 353, No. 3, 1251-1278 (2001).

[HN1] T. Haines, B. C. Ngô, Nearby cycles for local models of some Shimura varieties, Compositio Math. 133 (2002), 117-150.

[HN2] T. Haines, B.C. Ngô, Alcoves associated to special fibers of local models, Amer. J. Math. 124 (2002), 1125-1152.

[HP] T. Haines, A. Pettet, Formulae relating the Bernstein and Iwahori-Matsumoto presentations of an affine Hecke algebra, J. of Algebra 252 (2002), 127-149.

[KL2] D. Kazhdan, G. Lusztig, Schubert varieties and Poincaré duality, Proc. Symp. Pure Math. 36 (1980) 185-203.

[KW] R. Kiehl, R. Weissauer, Weil conjectures, Perverse Sheaves and $\ell$-adic Fourier transform, Springer Erg. Math. 3. Folge, 42 (2001).

[KR] R. Kottwitz, M. Rapoport, Minuscule alcoves for $G L_{n}$ and $G S p_{2 n}$, manuscripta math. 102 (2000), 403-428.

[RZ] M. Rapoport, Th. Zink, Über die lokale Zetafunktion von Shimuravarietäten. Monodromiefiltration und verschwindende Zyklen in ungleicher Charakteristik, Invent. Math. 68 (1982), 21-101.

Ulrich Görtz: Mathematisches Institut der Universität Bonn, Beringstr. 6, 53115 Bonn, Germany. Email: ugoertz@math.uni-bonn.de

Thomas J. Haines: Mathematics Department, University of Maryland, College Park, MD 20742-4015, USA. Email: tjh@math.umd.edu 